\title{
DIFFUSE AND LOCALISED VISUAL FIELD DEFECTS TO AUTOMATED PERIMETRY IN PRIMARY OPEN ANGLE GLAUCOMA
}

\author{
ERKAN MUTLUKAN \\ Detroit, Michigan
}

\begin{abstract}
SUMMARY
The occurrence of generalised or diffuse visual field depression in primary open angle glaucoma (POAG), in contrast to the presence of characteristic localised defects, is controversial. The frequency of diffuse visual field loss to automated static perimetry in the early stages of POAG was determined and compared with the frequency of localised defects. Twenty-five eyes of 25 consecutive POAG patients who met the selection criteria were tested on the Humphrey Visual Field Analyser with the Central 30-2 Threshold Test. Patients' mean age was 68 years. All eyes had visual acuity better than 6/9 with correction, refractive error of less than \pm 7 dioptres, no media opacities and normal pupils (3-6 mm). For all eyes, the frequency of abnormality on the STATPAC Total Deviation (TD) and Pattern Deviation (PD) plots were determined for all individual test points. Mean values and standard deviations were compared. The TD plots represent a composite of both diffuse and localised visual field depression, while PD plots are intended to reflect localised field defects. The frequency of involvement of the test points on the TD plots was higher than on the PD plots (mean \pm SD: $41.5 \pm 11.2 \%$ vs $27.5 \pm 10.9 \%$ ) for all presenting defects. When only deep defects (STATPAC $p<1 \%$ and $p<0.5 \%$ ) were evaluated, TD involvement was still more frequent than the PD (20.1 $\pm 9.4 \%$ vs $13.2 \pm 7.2 \%)$. The topographical pointwise incidence of pure generalised sensitivity loss in the visual field was less frequent when only deep defects were taken into consideration $(6.9 \pm 5.7 \%)$, and the incidence increased progressively with the inclusion of intermediate and shallow field defects $(9.6 \pm 6.8 \%$ and $14.0 \pm 8.4 \%$ respectively). A component of diffuse sensitivity depression is present at all significant levels of visual field loss in glaucoma. This component of generalised loss decreases as the depth of the field defects increases. This finding suggests that early
\end{abstract}

Correspondence to: E. Mutlukan, MD, PhD, Department of Ophthalmology, Henry Ford Health Sciences Center, 2799 West Grand Boulevard, Detroit, MI 48202, USA. Fax: 313-876 1327. diffuse field loss converts into well-defined pattern defects at later stages.

Generalised (diffuse) visual field loss found on automated static perimetry may be attributed to organic visual pathway disorder if there are no preretinal confounding factors such as uncorrected refractive error, media opacity or small pupil size.

The presence of generalised visual field loss found with Goldmann perimetry in primary open angle glaucoma $(\mathrm{POAG})^{1-5}$ was not observed in all patients. ${ }^{6,7}$ The only study claiming the absence of diffuse loss in POAG using automated perimetry (Competer) did not compare the visual fields of glaucomatous patients with the true age-corrected normal threshold values. ${ }^{8}$ Purely diffuse loss in the absence of other causes has recently been documented in POAG by Drance, ${ }^{9}$ using the Humphrey Visual Field Analyser's STATPAC empiric probability maps of Total Deviation (TD) versus Pattern Deviation (PD) maps in 3 eyes only. ${ }^{9}$ Therefore, the occurrence of generalised or diffuse visual field depression in POAG, in contrast to the presence of characteristic localised defects, remains controversial.

The objective of this study was to determine the frequency of diffuse visual field loss in the early stages of POAG and to compare this with the frequency of localised defects.

\section{MATERIALS AND METHOD}

Twenty-five eyes (17 right and 8 left) of 25 consecutive and perimetrically experienced patients (13 men and 12 women), aged between 35 and 82 years (mean 68 years), with previous clinical diagnosis of POAG were included in the study. The disease criteria in the study group were reproducible nerve fibre layer type visual field defects characteristic of glaucoma associated with grade 3-4 iridocorneal angle, untreated intraocular pressure greater 
than $21 \mathrm{mmHg}$ and/or cup-to-disc ratio greater than 0.6 . All eyes had $6 / 6$ or better acuity with correction less than \pm 7.00 dioptres spherical equivalent, no media opacities and normal (3-6 mm) pupils. Eyes with isolated relative scotomas (Aulhorn-Karmeyer classification, stage 1) and small absolute scotomas without and with connection to the blind spot (stage 2 and stage 3 respectively) were included. Glaucomatous eyes with large absolute scotomas extending to hemifield size or involving fixation (stages 4 and 5) were excluded to limit the study group to the relatively earlier stages of glaucomatous field involvement. None of the patients suffered from non-glaucomatous ocular disorders or systemic disease.

Visual field testing was performed with the Humphrey Visual Field Analyser, using the Central 30-2 Threshold Test Program with STATPAC, Goldmann size III $\left(4 \mathrm{~mm}^{2}\right)$ stimuli and a stimulus duration of 0.2 seconds. The criteria for an abnormal result were three or more adjacent test locations with at least $5 \mathrm{~dB}$ loss or one location with at least $10 \mathrm{~dB}$ loss for relative scotomas, and $0 \mathrm{~dB}$ threshold at one or more test locations for absolute scotomas. For all eyes, the frequency of abnormality was determined for all individual test points on the STATPAC Total Deviation (TD) and Pattern Deviation (PD) plots. The empiric probability maps indicate even the most shallow significant deviation from normal, and also help categorise the abnormal points according the depth and statistical significance of the defects. The detailed description of calculation of probability maps is available elsewhere. ${ }^{10}$ Significance levels (i.e. $p$ values) on an empiric probability map indicate confidence intervals or how often the measured threshold values occur at given test locations in normal visual fields. The probability maps are accepted as aids to clinical interpretation ${ }^{11-13}$ and have also been used for research. ${ }^{14-16}$ The frequency of abnormality for each test point on the TD and PD plots is the percentage ratio of patients with abnormal values at each empiric probability level (i.e. $p<5 \%, p<2 \%, p<1 \%$ and $p<0.5 \%$ ) at that point over the total number of eyes (i.e. 25). Mean values and standard deviations for frequency of abnormality at all test coordinates for both TD and PD plots were calculated at each empiric probability level. The numerical results for the TD and PD plots were then compared. The TD plots represent a composite of diffuse and localised visual field depression while PD plots reflect localised field defects. ${ }^{17,18}$ The difference, therefore, forms the basis of the study in that the difference between the TD and PD plots represents diffuse visual field loss.

\section{RESULTS}

All eyes had field abnormalities according to the criteria described above. STATPAC evaluation of the decibel threshold values revealed relative scotomas in 11 eyes (Aulhorn-Karmeyer classification, stage 1), isolated small absolute scotomas in 10 eyes (stage 2) and absolute scotomas connected to the blind spot in 4 eyes (stage 3 ) with the mean global field indices of $-5.2 \mathrm{~dB}$ Mean Deviation, 6.1 dB Pattern Standard Deviation, 5.4 dB Corrected Standard Pattern Deviation and 2.2 dB Short Term Fluctuation. The reliability indices from all test results were within the normal range (that is Fixation Losses $<20 \%$, False Positives and False Negatives $<33 \%$ ).

The cumulative results from the TD and PD plots demonstrated higher frequency of involvement in certain regions of the central visual field (superior and inferior Bjerrum area and above and below the nasal horizontal meridian), indicating preferential topographical distribution of glaucomatous scotomas, at all defect depths (Figs. 1, 2).

The frequency of involvement of the test points on the TD plots was higher than on the PD plots (mean $\pm \mathrm{SD}: 41.5 \pm 11.2 \%$ vs $27.5 \pm 10.9 \%, p<0.001$;

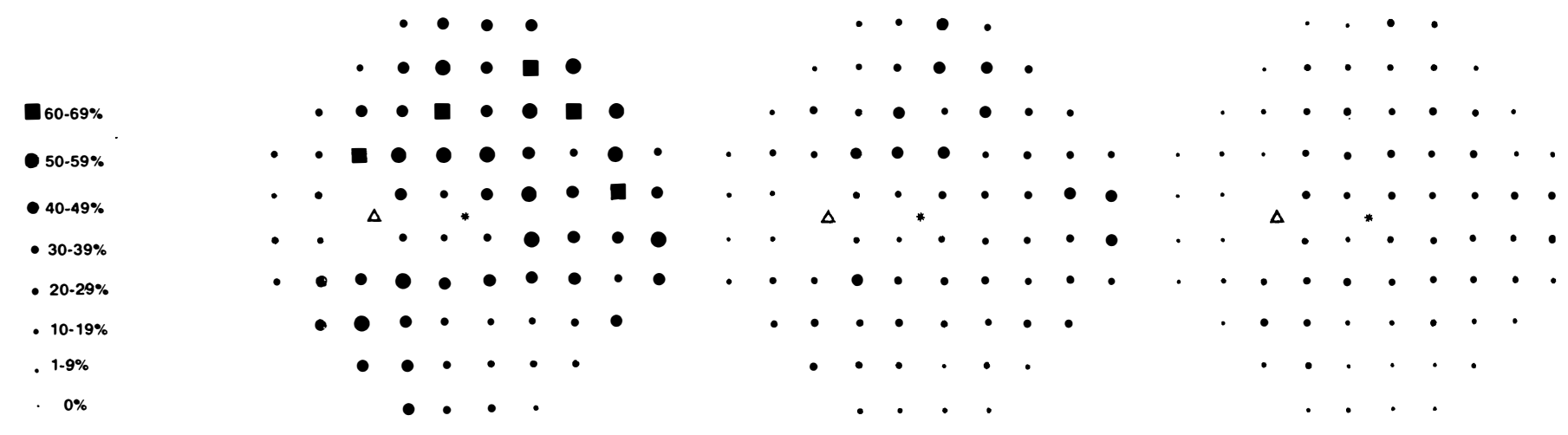

Fig. 1. Topographical distribution of glaucomatous field defects on the Total Deviation (TD) plots according to their confidence levels, as all defects on the left, intermediate and deep defects in the middle and deep defects on the right. Larger spots indicate higher frequency of involvement at individual test locations. 


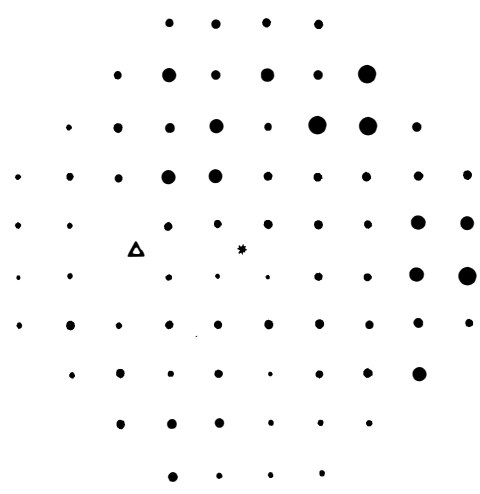

OUTSDE 95\% CONFDENCE NTERVAL

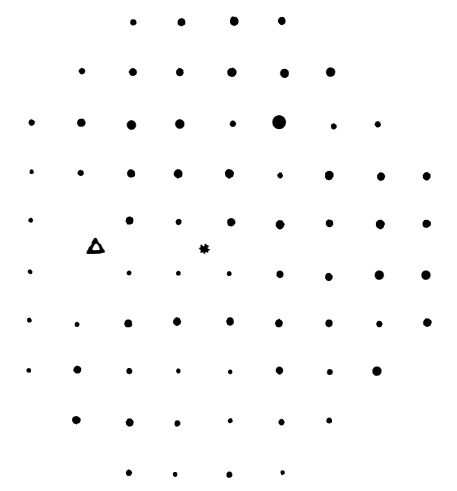

OUTSIDE 98\% CONFIDENCE WTERVAL

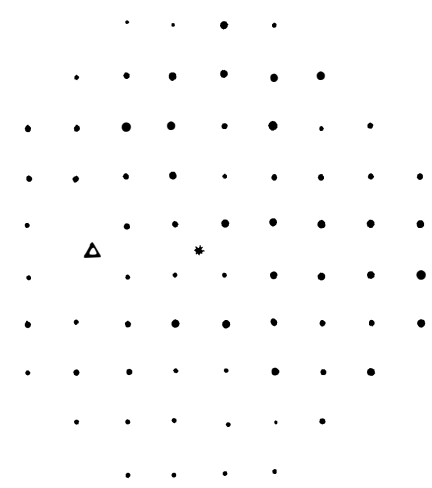

Fig. 2. Topographical distribution of glaucomatous field defects on the Pattern Deviation (PD) plots according to their confidence levels, as all defects on the left, intermediate and deep defects in the middle and deep defects on the right. Larger spots indicate higher frequency of involvement at individual test locations. In comparison with the TD plots of Fig. 1, the frequency of abnormality at individual test points was lower on PD plots.

Wilcoxon signed rank test) for all levels of defect (i.e. beyond $95 \%, 98 \%$ and $99 \%$ confidence intervals) (Figs. 1, 2). After shallow defects within the $98 \%$ confidence interval (i.e. $p<5 \%$ ) were excluded, the test points still had more frequent involvement on TD than on PD $(28.3 \pm 9.9 \%$ vs $18.7 \pm 8.4 \%$, $p<0.001)$. When only deep defects beyond the $99 \%$ confidence interval (STATPAC $p<1 \%$ and $p<0.5 \%$ ) were considered, TD involvement was still more frequent than the PD $(20.1 \pm 9.4 \%$ vs $13.2 \pm 7.2 \%$, $p<0.001)$. These results indicate the presence of a diffuse loss component along with localised defects (scotomas) of various depth. The frequency of generalised (diffuse) sensitivity loss was smallest when only deep defects (i.e. $p<1 \%$ and $p<0.5 \%$ ) were taken into consideration $(6.9 \pm 5.7 \%)$, and the frequency of diffuse loss increased significantly with the inclusion of intermediate (i.e. $p<2 \%, 9.6 \pm 6.8 \%$, $p=0.003$ ) and shallow field defects (i.e. $p<5 \% ; 14.0$ $\pm 8.4 \%, p<0.001)$.

\section{DISCUSSION}

In this study, the diffuse (generalised) sensitivity loss component of visual field loss in POAG was found to be present at various defect depths in the absence of other pre-retinal factors, using the STATPAC empiric probability maps of TD and PD. Also, various levels of the field loss caused by POAG were found to occur more frequently at certain areas.

The topographical selectivity with glaucomatous visual field loss has previously been reported for manual and automated conventional perimetric techniques. ${ }^{1,19-29}$ The frequency distribution of glaucomatous defects in the central visual field according to the defect depth and significance, however, has not been established before. As in previous studies, the cumulative STATPAC results from POAG patients in this study revealed visual field areas which were involved more frequently than elsewhere in the field. The results suggest that, when screening for glaucomatous defects using automated perimetry, the number of test locations may be reduced and optimised to concentrate on the most frequently involved areas. This strategey would achieve better patient compliance and shorter test duration. The preferential topographical involvement with localised 'glaucomatous' scotomas in the central visual field occurred concurrently with a component of diffuse loss at all levels of defect depths studied. This finding confirms the previous observations using kinetic Goldmann perimetry. ${ }^{27}$ Although 'pure diffuse loss' with complete absence of pattern defects may be rare in glaucoma ${ }^{30}$ this study clearly establishes that the generalised sensitivity loss component occurs along with the pattern scotomas. It also appears that the diffuse component tends to be less frequent with increasing glaucomatous defect depth, as illustrated in representative results from eyes with advanced defects (PSD $>10$ $\mathrm{dB}$ ) and almost identical TD and PD plots (Fig. 3). Therefore, it seems possible that the progression of glaucoma may involve conversion of a diffuse loss component into well-defined pattern defects in the central visual field. A prospective study with larger sample size rather than this cross-sectional study is more appropriate and necessary to reach a conclusion in that respect. The above findings indicate, however, that the evaluation of Total Deviation plots may facilitate the recognition of onset and progression of the disease in follow-up of glaucoma suspects and eyes with early glaucomatous field defects. In conclusion, a component of diffuse sensitivity depression is present at all significance levels of visual field loss in primary open angle glaucoma which affects selectively certain parts of the central visual field. 


\section{TOTAL DEVIATION}
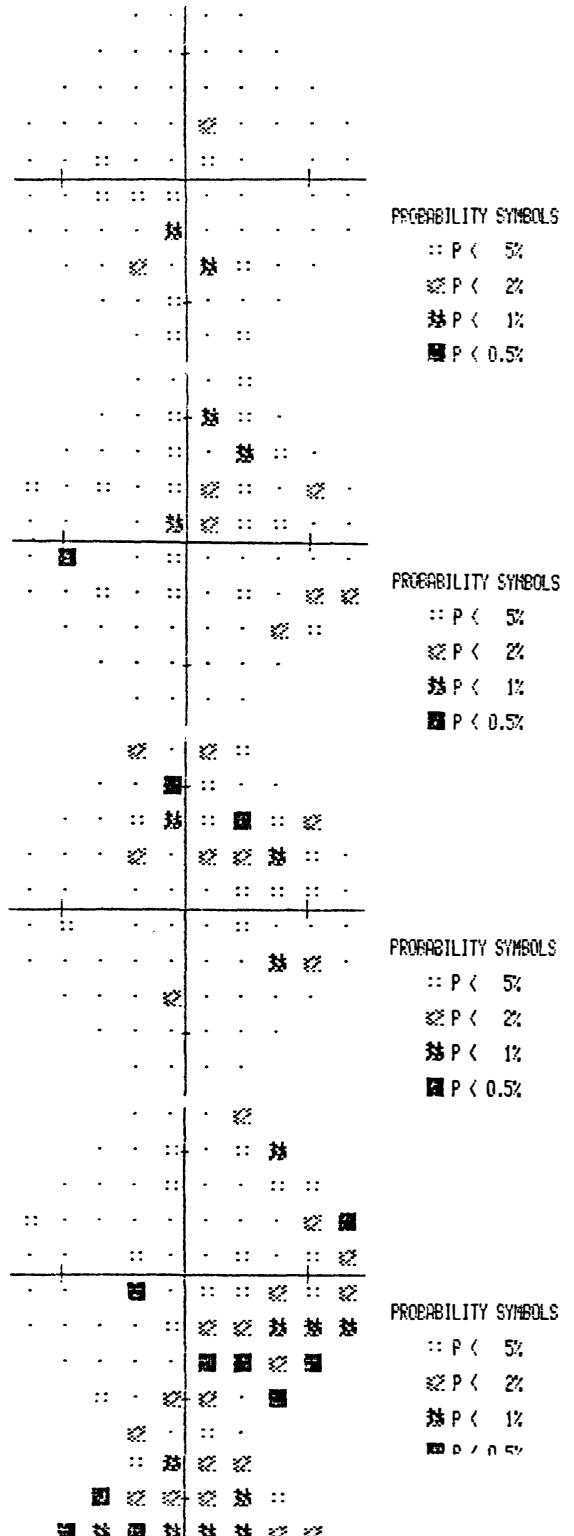

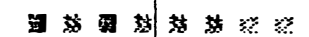

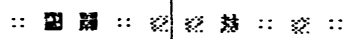

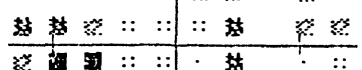

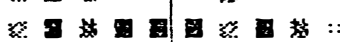

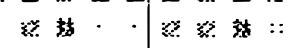

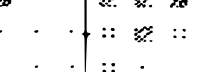

为

植

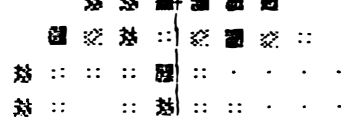

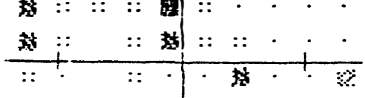

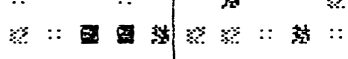

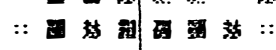

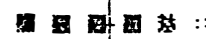

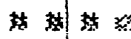

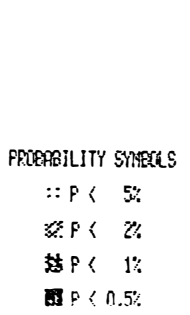

FMEAGILITY STITAS

$:: P \leqslant 5:$

\%P< $\%$

S $P<1 \%$

mo 0

\section{PATTERN DEVIATION}
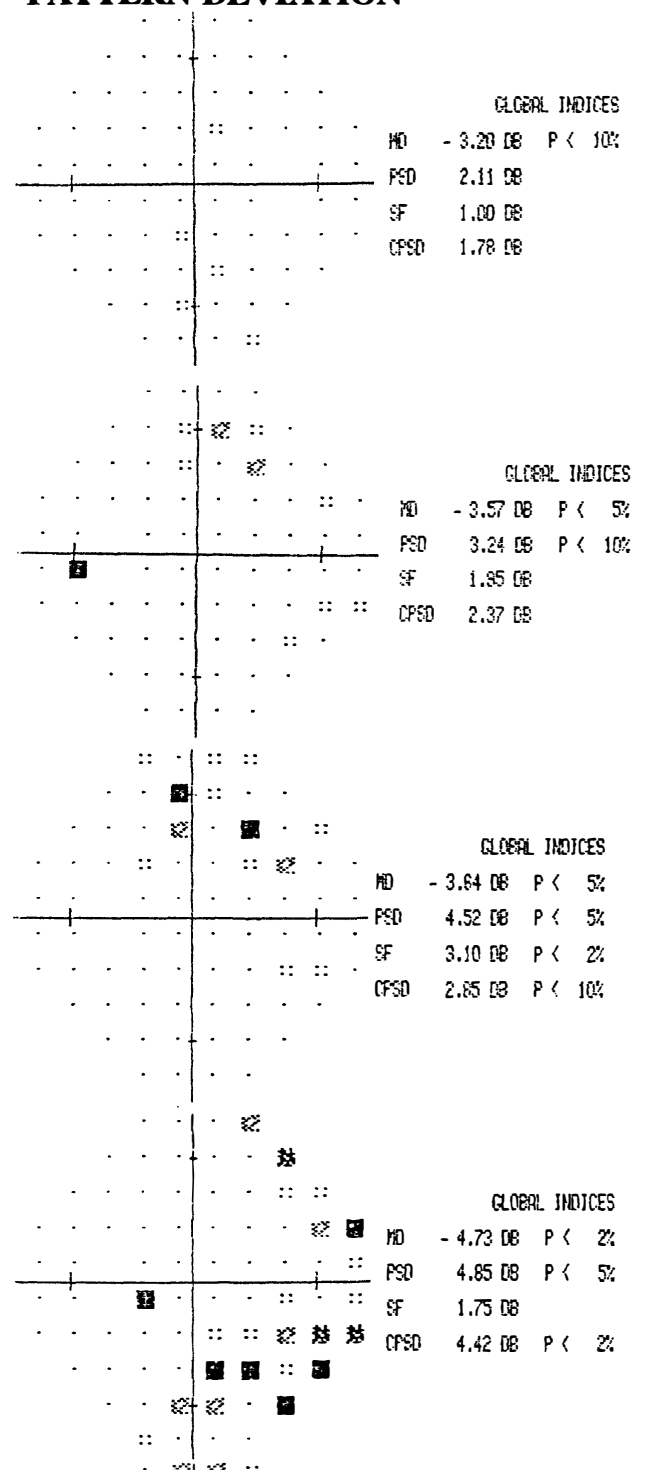

\%: : :

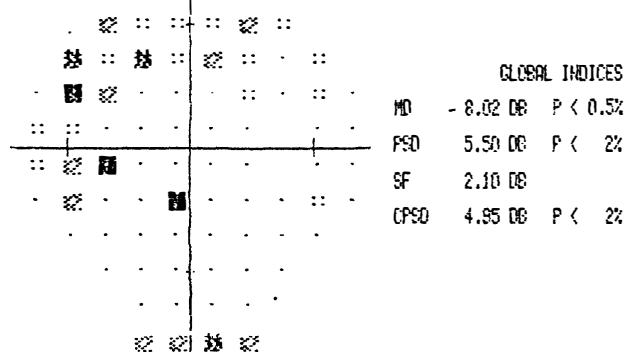

\%

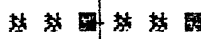

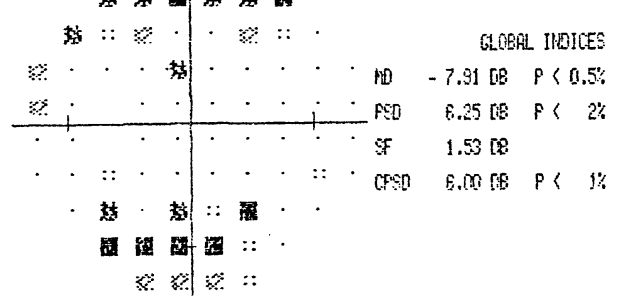

Fig. 3. Examples from Humphrey Visual Field Analyser STATPAC test results in 12 glaucomatous eyes. The single test results from these eyes are arranged in order of severity of field loss which increases from top to bottom as indicated by increasing Pattern Standard Deviation (PSD) scores. More advanced field deficits tended to have less difference between the $T D$ and PD plots as shown in eyes with a PSD score larger than $8 \mathrm{~dB}$. This may reflect decreasing frequency of generalised loss component with progression of scotomas. 


\section{TOTAL DEVIATION}

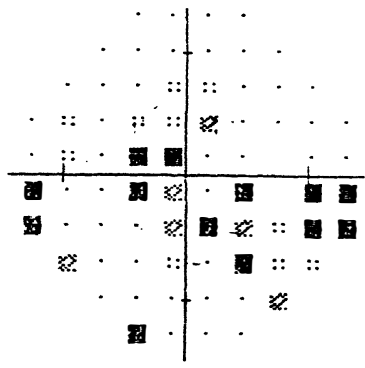

$\cdot:$
$::$

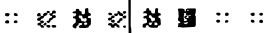

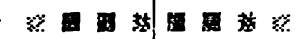

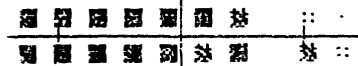

\%

ma日 :

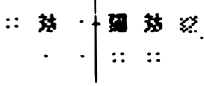

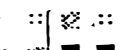

$::$ 过

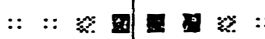

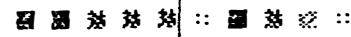

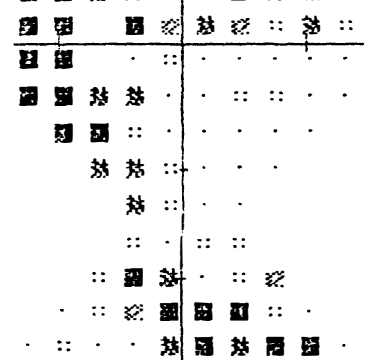

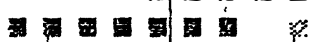

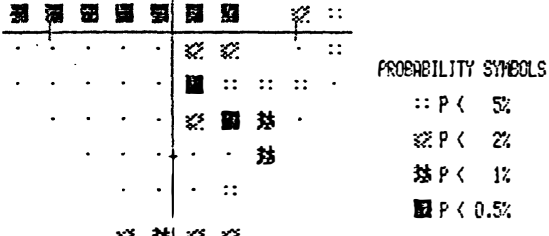

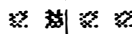

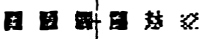

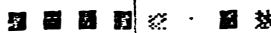

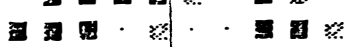

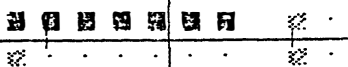

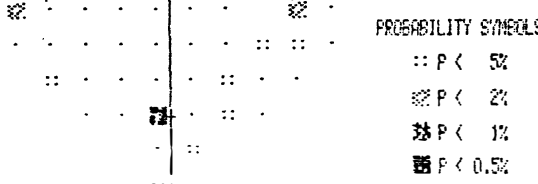

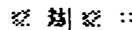

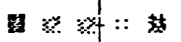

田

田国和国田

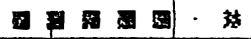

周望

部

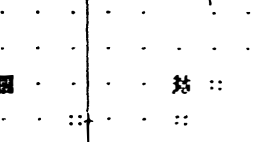

FOCEABILITY SIHEYLS

$:: \beta<5 \%$

$ख P<\%$

P $P<1 \%$

$B P<0.5 \%$

PRTERBLITY STERLS

$:: P<5 \%$

*⿻P $P<\%$

$P<1 \%$

$P<<0.5 \%$

PREREILITY SATERS

$:: P<5$

NF< 2

$P<1 \%$

EF $<0$

国 $P<0.5 \%$
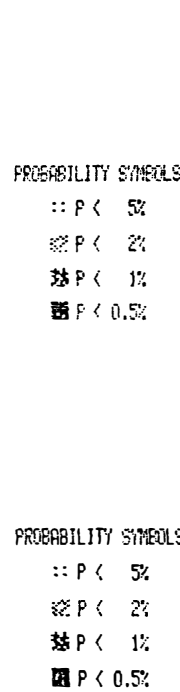

\section{PATTERN DEVIATION}
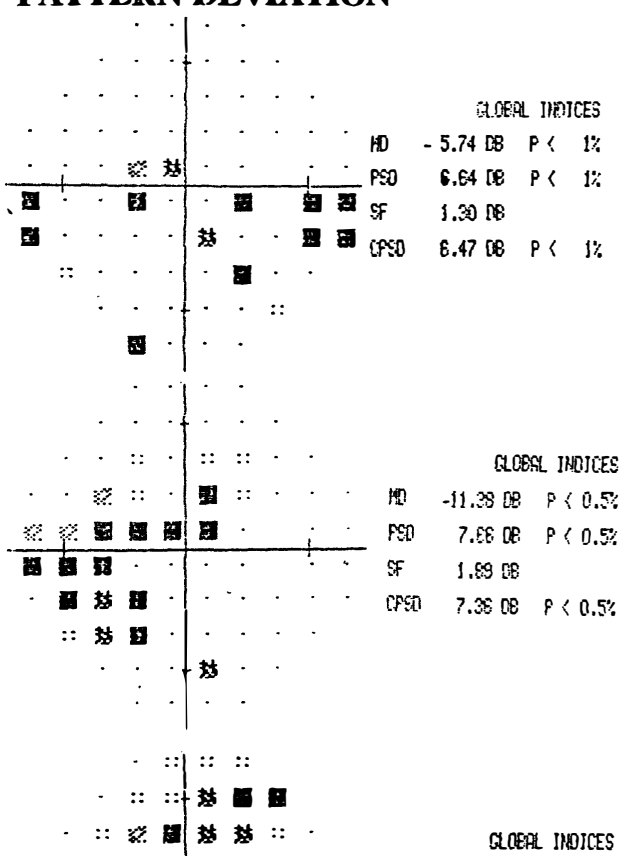

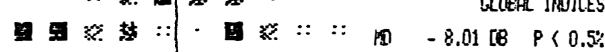

* $9 . \therefore \cdot+\cdot$.

1.

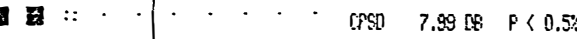

보

象 :

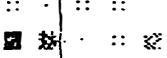

10

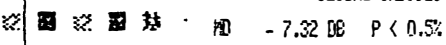

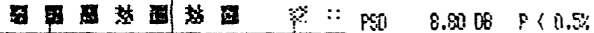

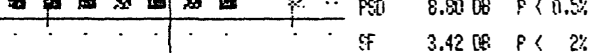

स 7.90 Le $P<0.5$

$*$

《济

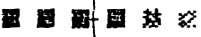

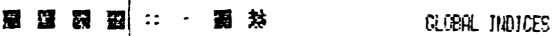

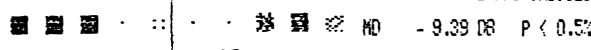

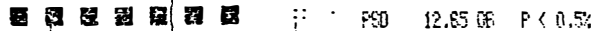

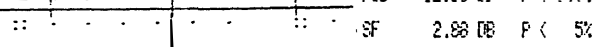

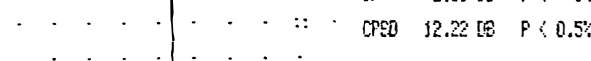
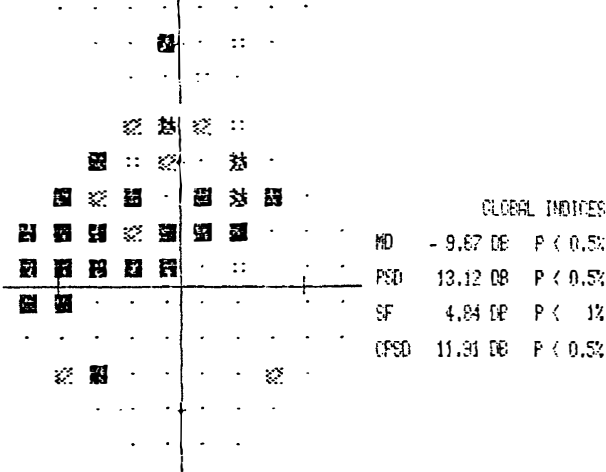

Fig. 3 (continued). 
This study was conducted at the Tennent Institute of Ophthalmology, University of Glasgow, with financial support from the International Glaucoma Association and the Royal National Institute for the Blind.

Key words: Glaucoma, Perimetry, Primary open angle glaucoma.

\section{REFERENCES}

1. Aulhorn E, Harms H. Early visual field defects in glaucoma. In: glaucoma symposium. Basel: Karger, 1967:151-86.

2. Armaly MF. The visual field defect and ocular pressure level in open angle glaucoma. Invest Ophthalmol 1969;8:105-24.

3. Armaly MF. Ocular pressure and visual fields: a 10year follow-up study. Arch Ophthalmol 1969;81:25-40.

4. Drance SM, Douglas GR, Airaksinen PJ, Schulzer M, Hitchings RA. Diffuse visual field loss in chronic openangle and low-tension glaucoma. Am J Ophthalmol 1987;104:577-80.

5. Anctil JL, Anderson DR. Early foveal involvement and generalised depression of the visual field in glaucoma. Arch Ophthalmol 1984;102:363-70.

6. Werner EB, Saheb N, Patel S. Lack of generalised constriction of affected visual field in glaucoma patients with visual field defects in one eye. Can J Ophthalmol 1982;17:53-5.

7. Langerhorst CT, van den Berg TJ, Greve EL. Is there general reduction of sensitivity in glaucoma? Int Ophthalmol 1989;13:31-5.

8. Heijl A. Lack of diffuse loss of differential light sensitivity in early glaucoma. Acta Ophthalmol (Copenh) 1989;67:353-60.

9. Drance SM. Diffuse visual field loss in open-angle glaucoma. Ophthalmology 1991;98:1533-8.

10. Asman P. Computer assisted interpretation of visual fields in glaucoma. Acta Ophthalmol 1992;70(Suppl 206):1-47.

11. Lieberman MF, Ewing RH. Reassessing split fixation in advanced glaucoma. In: Mills RP, Heijl A, editors. Perimetry update 1990/91. Amsterdam: Kugler \& Ghedini, 1991: 473-89.

12. Sommer A, Katz J, Quigley HA, et al. Clinically detectable nerve fibre atrophy precedes the onset of glaucomatous field loss. Arch Ophthalmol 1991; 109:77-83.

13. Anderson DR. Automatic static perimetry. St Louis: Mosby,1992:91-161.

14. Zamber R, Mills RP. Peripheral vs central confirma- tory testing. In: Heijl A, editor. Perimetry Update 1988/89. Amsterdam: Kugler \& Ghedini, 1989:409-16.

15. Katz J, Sommer A, Gaasterland DE, Anderson DR. Comparison of analytic algorithms for detecting glaucomatous visual field loss. Arch Ophthalmol 1991;109:1684-9.

16. Suzumura H, Endo N, Harasawa K, Suzuki H, Murao T. A new screening program with the Kowa automated perimeter: a peripheral isoptometry and central threezone program. In: Mills R, editor. Perimetry update 1992/93. Amsterdam: Kugler \& Ghedini, 1993:331-8.

17. Heijl A, Asman P. A clinical study of perimetric probability maps. Arch Ophthalmol 1989;107:199-203.

18. Heijl A, Lindgren G, Olsson J, Asman P. Visual field interpretation with empiric probability maps. Arch Ophthalmol 1989;107:204-8.

19. Drance SM. The early field defects in glaucoma. Invest Ophthalmol 1969;8:84-91.

20. Drance SM. The glaucomatous visual field. Invest Ophthalmol Vis Sci 1972;11:85-97.

21. Greve EL, Verduin WM. Detection of early glaucomatous damage. I. Visual field examination. Doc Ophthalmol Proc Ser 1977;14:103-14.

22. Rabin S, Kolesar P. Mathematical optimisation of glaucoma visual field screening protocols. Doc Ophthalmol 1978;45:361-80.

23. Kosaki H. The earliest visual field defect (IIa stage) in glaucoma by kinetic perimetry. Doc Ophthalmol Proc Ser 1979;19:255-9.

24. Fruno F, Matsuo H. Early stage progression in glaucomatous visual field changes. Doc Ophthalmol Proc Ser 1979;19:247-53.

25. Coughlan M, Friedman AI. The frequency distribution of early visual field defects in glaucoma. Doc Ophthalmol Proc Ser 1981;26:345-9.

26. Krieglstein GK, Schems W, Gramer E, Leydhecker W. Detectability of early glaucomatous field defects. Doc Ophthalmol Proc Ser 1981;26:19-24.

27. Hart WM Jr, Becker B. The onset and evolution of glaucomatous visual field defects. Ophthalmology 1982;89:268-79.

28. Heijl A, Lundqvist L. The frequency distribution of earliest glaucomatous visual field defects documented by automatic perimetry. Acta Ophthalmol (Copenh) 1984;62:658-64.

29. Henson DB, Chauhan BC. Informational content of visual field location in glaucoma. Doc Ophthalmol 1985;59:341-52.

30. Asman P, Heijl A. Diffuse visual field loss and glaucoma. Acta Ophthalmol (Copenh) 1994; 72:303-8. 